CONGENITAL HEART DISEASE

\title{
Poor sensitivity of routine fetal anomaly ultrasound screening for antenatal detection of atrioventricular septal defect
}

\author{
H ter Heide, J D R Thomson, G A Wharton, J L Gibbs
}

Heart 2004;90:916-917. doi: 10.1136/hrt.2003.018895

See end of article for
authors' affiliations
$\ldots \ldots \ldots \ldots . . . . . . . . . . . .$.
Correspondence to:
Dr John D Thomson,
Department of Paediatric
Cardiology, E Floor, Leeds
General Infirmary,
Calverley Street, Leeds
LS1 3EX, UK;
john.thomson@lineone.net
Accepted
26 November 2003

\begin{abstract}
Objective: To report the antenatal detection rate in a consecutive series of liveborn infants with atrioventricular septal defect (AVSD).

Design: Review and analysis of referrals for detailed fetal echocardiography and postnatal diagnosis of AVSD.

Setting: Tertiary referral centre for congenital heart disease centre with data prospectively collected between 1996 to 2001

Results: 92 consecutively liveborn infants with AVSDs were identified of which 27 (29\%) were detected by routine obstetric antenatal ultrasound. The antenatal diagnosis rate was worse for liveborn infants with trisomy 21 (12 of $49(25 \%)$ v 15 of $43(35 \%)$ chromosomally normal children) and for infants with AVSD without other structural heart disease (18 of $74(24 \%) \vee 9$ of $18(50 \%)$ infants with associated structural heart disease).

Conclusion: Despite the potential ability of fetal ultrasound to detect AVSDs, the antenatal diagnosis rate is poor. This is particularly true for infants with trisomy 21 and is of importance when counselling parents with an apparently normal fetal ultrasound scan.
\end{abstract}

$\mathrm{P}$ renatal screening for major congenital heart disease is now a routine part of obstetric ultrasound anomaly screening in most obstetric centres in the UK. The four chamber view was first introduced into routine obstetric screening in 1986 as a method of identifying major cardiac malformations, the majority of which occur in women with no identifiable risk factors. ${ }^{12}$ Screening requires imaging of a four chamber view identifying a normal cardiac position, two atria and two ventricles of approximately equal size, and the two atrioventricular valves forming an offset cross at the centre of the heart.

Prenatal diagnosis of atrioventricular septal defect (AVSD) is particularly important because of its association with chromosomal abnormalities (especially trisomy 21), as well as with additional serious cardiac and extracardiac abnormalities. Previous antenatal studies have shown AVSD to be among the most commonly detected cardiac lesions. ${ }^{3}$

Although some studies report excellent sensitivity for detection of congenital heart disease, they have concentrated on fetal rather than liveborn cohorts..$^{3-5}$ We report a low rate of antenatal diagnosis of AVSD in a consecutive series of infants born alive with the defect.

The congenital heart disease department in Leeds is a tertiary referral centre for congenital heart disease serving a population of about 5.25 million in Yorkshire, Humberside, and Northern Trent. There are stable referral patterns with no other centres in the region providing surgery for congenital heart disease or detailed fetal or paediatric echocardiography. A fetal cardiac ultrasound training programme was started in 1989 and was taken up by 19 of the 22 obstetric units within the catchment area. Our aim in this study was to assess the antenatal diagnosis rate in a cohort of children born alive with AVSD between 1996 and 2001.

\section{PATIENTS AND METHODS}

All patients with AVSD born between 1996 and 2001 within the catchment region of the tertiary centre were identified by a search of prospectively collected, comprehensive diagnostic information held in the department's electronic congenital heart disease database.

Information relating to referrals for detailed fetal echocardiography was retrieved from the same database. Obstetric ultrasound screening was performed in referring hospitals according to unit specific protocols. A four chamber view of the heart was included as part of routine fetal anomaly screening scan in all but a single unit (responsible for $1.5 \%$ of all live births in the region) in which no prenatal ultrasound screening was offered at all. None of the mothers of the children making up our study population were booked at this unit and all therefore received screening. The Leeds fetal echocardiography team provided formal, on-site training in fetal cardiac ultrasonography to 19 of the referring obstetric teams.

Detailed fetal echocardiography was offered to all patients with abnormal, possibly abnormal, or difficult four chamber views, as well as to patients with a family history of congenital heart disease, soft markers for aneuploidy, or extracardiac malformations in the fetus.

\section{Statistical analysis}

Fisher's exact test was used to compare observed frequencies between groups.

\section{RESULTS}

Ninety two liveborn children with AVSDs were identified. Sixty four $(69 \%)$ had complete defects, 11 (12\%) a partial defect, and 17 (18\%) an AVSD in association with complex congenital heart disease (table 1). Down's syndrome was diagnosed in 49 children (53\%).

We were unable to trace antenatal details for two (2.1\%) patients in our study group; for these two children we assumed that there was no antenatal diagnosis of an AVSD.

Twenty seven of 92 cases of AVSD among liveborn infants (29.3\%, 95\% confidence interval (CI) 18.4 to 35.6) were 
Table 1 Associated cardiac lesions

\begin{tabular}{ll}
\hline Anatomy & Number (\%) \\
\hline Right atrial isomerism* & $6(33 \%)$ \\
Left atrial isomerism* & $3(17 \%)$ \\
Tetralogy of Fallot & $3(17 \%)$ \\
Pulmonary atresia & $3(17 \%)$ \\
Coarctation & $1(6 \%)$ \\
Double outlet right ventricle & $1(6 \%)$ \\
Muscular ventricular septal defect & $1(6 \%)$ \\
\hline \multirow{2}{*}{ *All with complex intracardiac abnormalities. } &
\end{tabular}

detected by routine antenatal obstetric ultrasound screening. All patients with antenatally diagnosed AVSDs had been referred for detailed fetal echocardiography. In one case where a four chamber view of the fetal heart could not be obtained at screening, specialist fetal echocardiography also failed to evaluate cardiac anatomy because of gross maternal obesity and an AVSD was diagnosed postnatally.

Nine of $18(50 \%, 95 \%$ CI 4.8 to 13.2) infants with AVSD in association with complex congenital heart disease were identified by routine antenatal screening.

In the 49 liveborn infants with AVSD and Down's syndrome the AVSD (and trisomy 21) was diagnosed antenatally in only $12(24.5 \%, 95 \%$ CI 6.1 to 17.9$)$. In the 43 children born with an AVSD and normal chromosomes the cardiac abnormality was detected antenatally in 15 (34.9\%, 95\% CI 8.8 to 21.1; not significant).

\section{DISCUSSION}

Antenatal diagnosis of AVSD is of particular clinical and medicolegal importance because of its strong association with trisomy 21. Despite multiple potential anomalies in babies with trisomy 2l, non-cardiac sonographic markers (for example, renal pelvic dilatation) are often subtle and are frequently missed during screening. Therefore, the ability of fetal ultrasound to detect congenital heart disease accurately in this setting is particularly important. ${ }^{16}$ There is an understandable and widely held view that AVSD, particularly in the presence of a chromosomal anomaly, should not be missed at a screening scan. In low risk fetal populations, however, detection rates during screening for congenital heart disease as a whole are reported to vary from 13$92 \%{ }^{478}$ The antenatal detection rate for a consecutive series of liveborn patients with AVSD, a lesion potentially diagnosable on the standard four chamber view, has not been reported before. In our series $70 \%$ of AVSDs were diagnosed only after birth despite the majority of referring centres in our study having received basic on-site fetal echocardiography training. Although not significant, the rate of detection in babies with trisomy 21 was less than for chromosomally normal infants.
Once complex cases known to be associated with AVSDs in infants without trisomy ${ }^{9}$ were removed from the analysis the detection rate was similar at $25 \%$ and $23 \%$ for infants with and without trisomy 21 , respectively.

It is beyond the scope of this paper to discuss detection rates for other congenital cardiac lesions; however, the echocardiographic hallmark of the AVSD is a common atrioventricular valve, which even on a good four chamber view can appear superficially normal.

By implication AVSDs may be more difficult to detect at standard screening than lesions with more obvious structural pathology. Although training for obstetric screening units was almost universal in this series and this undoubtedly translated to a greater awareness of congenital heart disease, perhaps technical proficiency can be gained only by exposure to a large number of abnormal cases. For the average obstetric screening unit this is an unrealistic expectation. Effective screening for this important lesion may require a different strategy.

\section{Conclusion}

The antenatal detection rate for AVSD by standard ultrasound screening in the four chamber view is poor overall, with a trend towards being even poorer in the presence of trisomy 21.

\section{Authors' affiliations}

H ter Heide, J D R Thomson, G A Wharton, J L Gibbs, Department of Paediatric Cardiology, Leeds General Infirmary, Leeds, UK

There were no conflicts of interest. All authors had full access to all the data in the study. The corresponding author had the final responsibility for the decision to submit for publication.

\section{REFERENCES}

1 Allan LD, Crawford DC, Chita SK, et al. Prenatal screening for congenital heart disease. BMJ 1986;292:1717-9.

2 Tegnander E, Eik-Nes SH, Linker DT. Incorporating the four chamber view of the fetal heart into the second trimester routine fetal examination. Ultrasound Obstet Gynecol 1994:4:24-8.

3 Allan LD, Sharland GK, Milburn A, et al. Prospective diagnosis in 1,006 consecutive cases of congenital heart disease in the fetus. J Am Coll Cardiol 1994;23:1452-8.

4 Achiron R, Glaser J, Gelernter I, et al. Extended fetal echocardiographic examination for detecting cardiac malformations in low risk pregnancies. BMJ 1992;304:671-4

5 Copel JA, Pilu G, Green J, et al. Fetal echocardiographic screening for congenital heart disease: the importance of the four-chamber view. Am J Obstet Gynecol 1987; 157:648-55.

6 Stumpflen I, Stumpflen A, Wimmer M, et al. Effect of detailed fetal echocardiography as part of routine prenatal ultrasonographic screening on detection of congenital heart disease. Lancet 1996;348:854-7

7 Stoll C, Alembik Y, Dott B, et al. Evaluation of prenatal diagnosis of congenital heart disease. Prenat Diagn 1998;18:801-7.

8 Allan LD. Atrioventricular septal defect in the fetus. Am J Obstet Gynecol 1999;181:1250-3.

9 Delisle MF, Sandor GGS, Tessier F. Outcome of fetuses diagnosed with atrioventricular septal defect. Obstet Gynaecol 1999;94:763-7. 